Annals of Pure and Applied Mathematics

Vol. 14, No. 1, 2017, 53-62

ISSN: 2279-087X (P), 2279-0888(online)

Published on 4 July 2017

Annals of

www.researchmathsci.org

DOI: http://dx.doi.org/10.22457/apam.v14n1a7

Pure and Applied

Mathematics

\title{
Interval Edge Coloring of Sudha Grid of Hexagons, Gear and Helm Graphs
}

\author{
S.Sudha ${ }^{1}$ and G.M.Raja ${ }^{2}$ \\ ${ }^{1}$ Ramanujan Institute for Advanced Study in Mathematic, University of Madras \\ Chennai-600005. ${ }^{1}$ E-mail: sudhasubramanian1955@gmail.com \\ Corresponding author. ${ }^{2} \mathrm{E}$-mail: rajagopalmalliga@gmail.com
}

Received 1 June 2017; accepted 30 June 2017

\begin{abstract}
An arbitrary non-empty finite subset of consecutive positive integers is called an interval in graph theory. An edge coloring of a graph $G$ with colors $1,2, \ldots k$ is called an $k$-interval edge coloring if all the colors are used so that the colors of the edges incident to any vertex of $G$ are distinct and are consecutive. The interval edge coloring of Sudha grid of hexagons, gear and Helm graphs are stated as theorems in this paper and we found their interval edge chromatic numbers to be $3, n$ and $n$ respectively.
\end{abstract}

Keywords: Interval edge coloring, Sudha grid of hexagons, gear graph, Helm graph

AMS Mathematics Subject Classification (2010): $05 \mathrm{C} 15$

\section{Introduction}

The concept of interval edge coloring was introduced by Asratian and Kamalian [1] in 1987 in their paper on interval edge colorings of a multigraph. Kamalian [5] gave the interval edge coloring of simple cycles in a cyclic way. Saha et al. [8] discussed an application of graph theory. Petrosyan [7] has discussed about the interval edge colorings of complete graphs and $n$ dimensional cubes. Sathis et al. [9] gave the dominator coloring of graphs. Kamalian [3] has obtained the interval coloring of complete bipartite graphs and trees. Kamalian [4] has also found the result on cyclically interval edge coloring of trees. Sudha grid graphs was introduced by Sudha et al. [10]. Sudha et al. [11] have found the interval edge coloring of grid graphs. Vaidya et al. [12] discussed the prime cordial labeling of wheel related graphs and Meena et al. [6] have found the prime labeling for some Helm related graphs. Kaliraj [2] found the equitable coloring of helm graph and gear graph. In this paper, we obtained the interval edge coloring of Sudha grid of hexagons $S_{h}(m, n)$, gear graph $G_{0,2 n}$ and Helm graph $H_{0,2 n}$.

2. Main reults

Definition 1. An edge coloring of a graph is the coloring of edge of the graph with the minimum number of colors without any two adjacent edges having the same color.

An edge coloring of a graph $G$ with colors $1,2, \ldots k$ is called an $k$-interval edge coloring if all the colors are used so that the colors of the edges incident to any vertex of $G$ are distinct and are consecutive. 


\section{S. Sudha and G. M. Raja}

The smallest integer $k$ for which the graph $G$ is $k$-interval edge coloring is known as the chromatic number of interval edge coloring of $G$ and is denoted by $\chi_{i e}(G)$.

Definition 2. Sudha grid of hexagons $S_{h}(m, n)$ is an induced subgraph of the strong product of two paths $P_{m}$ and $P_{n}(n$ is odd,$\geq 3$ and $m \equiv 0(\bmod 4))$ with the vertex set given by

and the edge set

$$
V\left(S_{h}(m, n)\right)=\left\{\left(u_{i}, v_{j}\right) / \begin{array}{c}
i \equiv 0,1(\bmod 4) \text { if } j \text { is even } \\
\text { otherwise } j \text { is odd }, 1 \leq i \leq n
\end{array}\right.
$$

$$
E\left(S_{h}(m, n)\right)=\left\{\begin{array}{c}
\left(u_{i}, v_{j}\right)\left(u_{k}, v_{l}\right) / u_{i} u_{k} \in E\left(P_{m}\right) \text { and } v_{j} v_{l} \in E\left(P_{n}\right) \\
; u_{i} u_{k} \in E\left(P_{m}\right) \text { and } j=l
\end{array}\right\}
$$

where $V\left(P_{m}\right)=\left\{u_{i} / 1 \leq i \leq m\right\}, V\left(P_{n}\right)=\left\{v_{j} / 1 \leq j \leq n\right\},\left(u_{i}, v_{j}\right)$ represents the vertex in the $i j^{\text {th }}$ position and $\left(u_{i}, v_{j}\right)\left(u_{k}, v_{l}\right)$ represents the edge joining the vertices at the $i j^{\text {th }}$ and $k l^{\text {th }}$ position of the strong product of $P_{m}$ and $P_{n}$.

Illustration 1. Sudha grid of hexagon $S_{h}(8,7)$ is a graph with the vertex set $\left\{v_{1,2}, v_{1,4}, v_{1,6}, v_{2,1}, v_{2,3} v_{2,5}, v_{2,7}, v_{2,1}, v_{3,3}, v_{3,5}, v_{3,7}, v_{4,2}, v_{4,4}, v_{4,6}, v_{5,2}, v_{5,4}, v_{5,6}\right.$, $\left.v_{6,1}, v_{6,3}, v_{6,5}, v_{6,7}, v_{7,1}, v_{7,3}, v_{7,5}, v_{7,7}, v_{8,2}, v_{8,4}, v_{8,6}\right\}$ as shown in figure 1 .

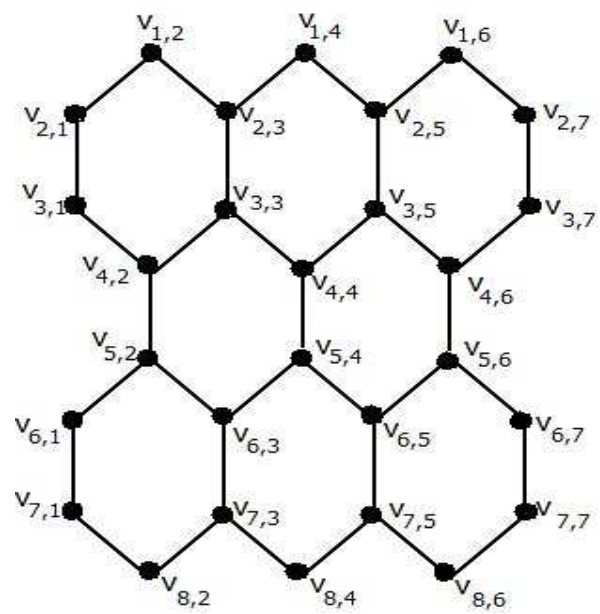

Figure 1: Sudha grid of hexagon $S_{h}(8,7)$

Definition 3. The gear graph $G_{0,2 n}$ is obtained from a wheel graph $W_{0, n}$ by subdividing the edges once of the outer cycle of the wheel $W_{0, n}$.

Illustration 2. $G_{0,8}$ is a gear graph with the vertex set $\left\{v_{0}, v_{1}, v_{2}, v_{3}, v_{4}, u_{1}, u_{2}, u_{3}, u_{4}\right\}$ and the edge set

$\left\{v_{0} v_{1}, v_{0} v_{2}, v_{0} v_{3}, v_{0} v_{4}, v_{1} u_{1}, u_{1} v_{2}, v_{2} u_{2}, u_{2} v_{3}, v_{3} u_{3}, u_{3} v_{4}, v_{4} u_{4}, u_{4} v_{1},\right\}$ as shown in figure 2 . 
Interval Edge Coloring of Sudha Grid of Hexagons, Gear and Helm Graphs

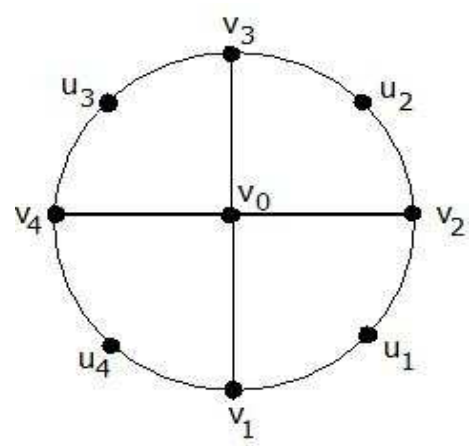

Figure 2: Gear graph $G_{0,8}$

Definition 4. The Helm graph $H_{0,2 n}$ is obtained from a wheel graph $w_{0, n}$ by adjoining an edge to the vertices of the outer cycle of the wheel $w_{0, n}$.

Illustration 3. $H_{0,8}$ is a Helm graph with the vertex set $\left\{v_{0}, v_{1}, v_{2}, v_{3}, v_{4}, u_{1}, u_{2}, u_{3}, u_{4}\right\}$ and the edge set

$\left\{v_{0} v_{1}, v_{0} v_{2}, v_{0} v_{3}, v_{0} v_{4}, v_{1} v_{2}, v_{2} v_{3}, v_{3} v_{4}, v_{4} v_{1}, v_{1} u_{1}, v_{2} u_{2}, v_{3} u_{3}, v_{4} u_{4}\right\}$ as shown in figure 3 .

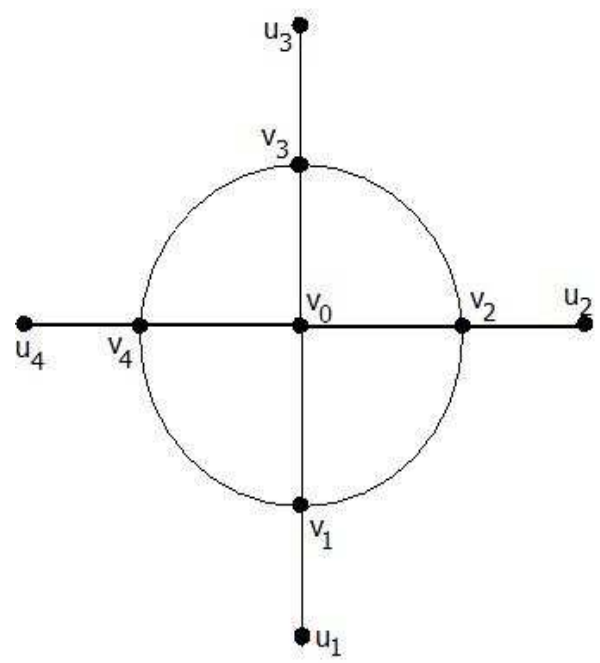

Figure 3: Helm graph $H_{0,8}$

Theorem 1. Sudha grid of hexagons $S_{h}(m, n)$ admit interval edge coloring and its chromatic number is 3 .

Proof: Sudha grid of hexagons $S_{h}(m, n)$ is the induced subgraph of the strong product of the path $P_{m}$ and the path $P_{n}$ (for odd $m \geq 3$ and $n \equiv 0(\bmod 4)$ ). The vertices of $S_{d}(m, n)$ are denoted by $v_{i, j}, 1 \leq i \leq n, 1 \leq j \leq m$ as shown in figure 4 . 
S. Sudha and G. M. Raja

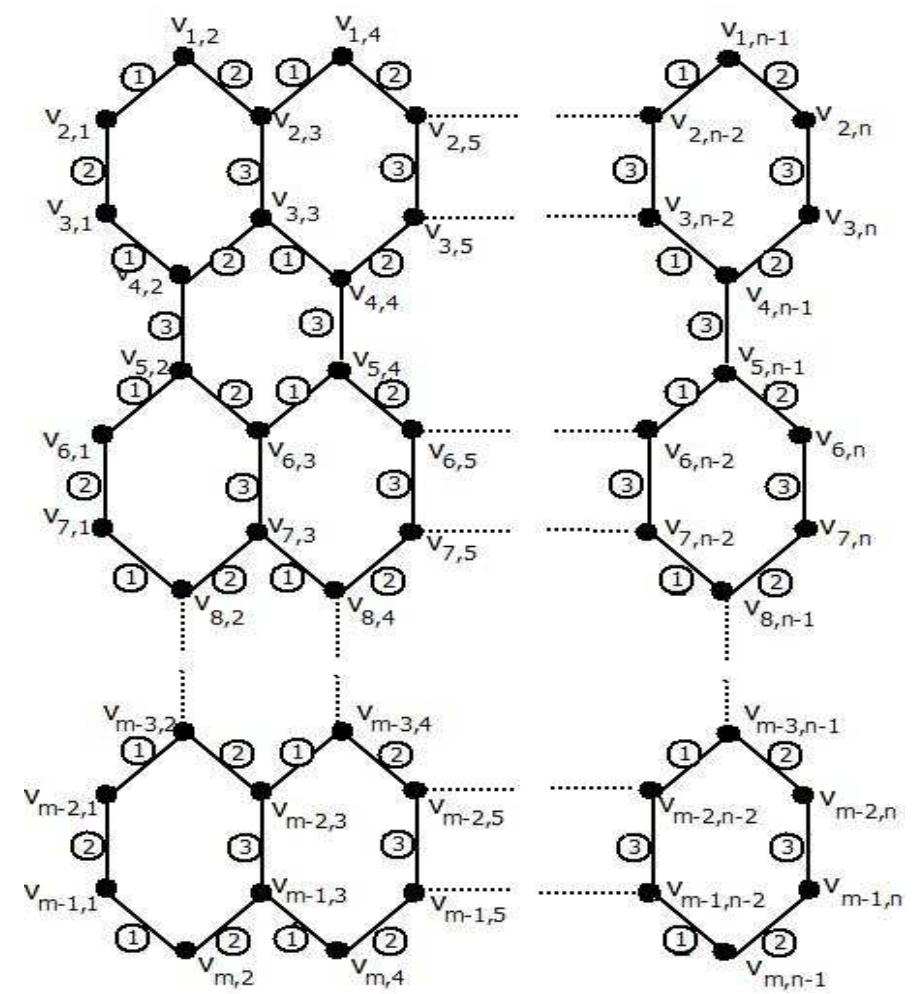

Figure 4: Sudha grid of hexagons $S_{h}(m, n)$

The function $f$ from the vertex set of $S_{h}(m, n)$ to the set of colors $\{1,2,3\}$ is defined as

$$
\begin{array}{r}
f\left(v_{i, j+1} v_{i+1, j}\right)=\left\{\begin{array}{l}
1, \quad i \equiv 1(\bmod 4) \\
2, \quad i \equiv 3(\bmod 4)
\end{array},\right. \\
f\left(v_{i, j} v_{i+1, j+1}\right)=\left\{\begin{array}{l}
1, i \equiv 3(\bmod 4) \\
2, i \equiv 1(\bmod 4)
\end{array}\right. \\
f\left(v_{i, j} v_{i+1, j}\right)=3, j \neq 1
\end{array}
$$

Sudha grid of hexagons $S_{h}(m, n)$ has interval edge coloring with this type of coloring and hence the interval edge chromatic number of $S_{h}(m, n)$ is 3 .

Illustration 4. Consider the graph $S_{h}(8,7)$. Using theorem 1 , we assign the color 1 to the edges $v_{1,2} v_{2,1}, v_{1,4} v_{2,3}, v_{1,6} v_{2,5}, v_{3,1} v_{4,2}, v_{3,3} v_{4,4}, v_{3,5} v_{4,6}, v_{5,2} v_{6,1}, v_{5,4} v_{6,3}$, $v_{5,6} v_{6,5}, v_{7,1} v_{8,2}, v_{7,3} v_{8,4}, v_{7,5} v_{8,6}$, the color 2 to the edges $v_{1,2} v_{2,3}, v_{1,4} v_{2,5}, v_{1,6} v_{2,7}, v_{2,1} v_{3,1}, v_{3,3} v_{4,2}, v_{3,5} v_{4,4}, v_{3,7} v_{4,6}, v_{5,2} v_{6,3}, v_{5,4} v_{6,5}$ $v_{5,6} v_{6,7}, v_{6,1} v_{7,1}, v_{7,3} v_{8,2}, v_{7,5} v_{8,4}, v_{7,7} v_{8,6}$ and the color 3 to the edges $v_{2,3} v_{3,3}, v_{2,5} v_{3,5}, v_{2,7} v_{3,7}, v_{4,2} v_{5,2}, v_{4,4} v_{5,4}, v_{4,6} v_{5,6}, v_{6,3} v_{7,3}, v_{6,5} v_{7,5}, v_{6,7} v_{7,7}$ as shown in figure 5 . 
Interval Edge Coloring of Sudha Grid of Hexagons, Gear and Helm Graphs

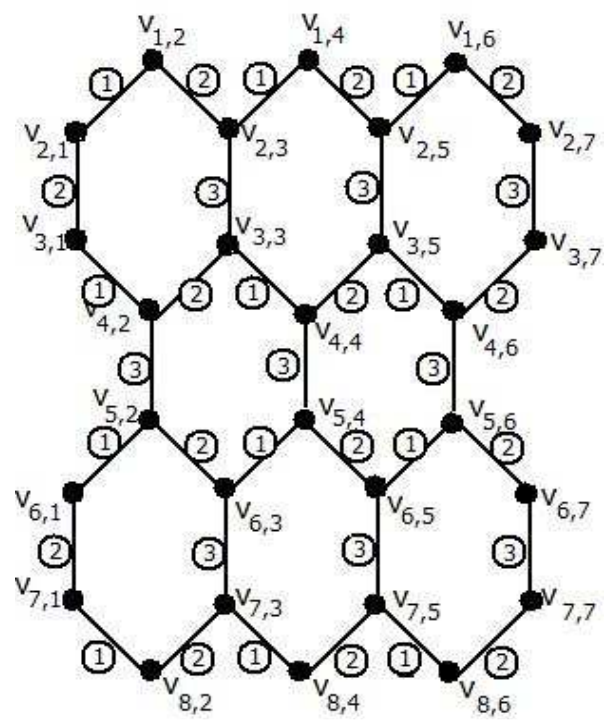

Figure 5: Sudha grid of hexagon $S_{h}(8,7)$

Hence, $\chi_{\text {ie }}\left(S_{h}(8,7)\right)=3$.

Theorem 2. The gear graph $G_{0,2 n}$ admits interval edge coloring and its chromatic number of interval edge coloring is $n$.

Proof: The gear graph $G_{0,2 n}$ has $2 n+1$ vertices and $3 n$ edges. The vertex set of $G_{0,2 n}$ $\operatorname{is} V\left(G_{0,2 n}\right)=\left\{v_{0}, v_{i}, u_{i} / 1 \leq i \leq n\right\}$ and the edge set of $G_{0,2 n}$ is

$E\left(G_{0,2 n}\right)=\left\{v_{0} v_{i} / 1 \leq i \leq n\right\} \cup\left\{v_{i} u_{i} / 1 \leq i \leq n\right\} \cup\left\{u_{i} v_{i+1} / 1 \leq i \leq n-1\right\} \cup u_{n} v_{1}$ as shown in figure 6.

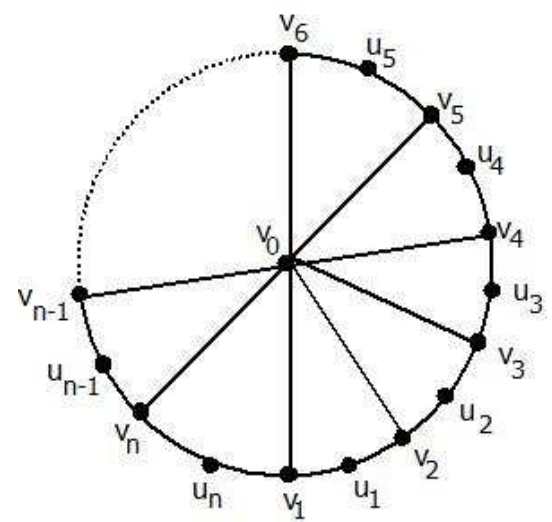

Figure 6: Gear graphs $G_{0,2 n}$

The function $f$ is defined as the coloring from the edges of $G_{0,2 n}$ to the set of colors (positive integers) $\{1,2,3, \ldots n\}$, as follows : 
If $n$ is odd,

$$
\begin{gathered}
\text { S. Sudha and G. M. Raja } \\
f\left(v_{0} v_{i}\right)=f\left(u_{i} v_{i+1}\right)=\left\{\begin{array}{cc}
2 i, & 1 \leq i \leq\left\lfloor\frac{n}{2}\right\rfloor \\
2(n-i)+1, & \left\lceil\frac{n}{2}\right\rfloor<i<n
\end{array}\right. \\
f\left(v_{0} v_{n}\right)=1, \\
f\left(u_{n} v_{1}\right)=3, \\
f\left(v_{i} u_{i}\right)=\left\{\begin{array}{cc}
2 i-1, & 1 \leq i \leq\left\lfloor\frac{n}{2}\right\rfloor \\
2(n-i)+2, & \left\lfloor\frac{n}{2}\right\rfloor+1<i \leq n
\end{array}\right. \\
f\left(v_{\left\lfloor\frac{n}{2}\right\rfloor+1} u_{\left\lfloor\frac{n}{2}\right\rfloor+1}\right)=n-2 .
\end{gathered}
$$

$$
\begin{gathered}
f\left(v_{0} v_{\left\lceil\frac{n}{2}\right\rceil}\right)=n, \\
\text { and } f\left(u_{\left\lceil\frac{n}{2}\right\rceil} v_{\left\lceil\frac{n}{2}\right\rceil+1}\right)=n-3 .
\end{gathered}
$$

With this type of coloring, the gear graph $G_{0,2 n}$ satisfies the definition of interval edge coloring and its chromatic number of interval edge coloring is $n$.

Illustration 5. Consider the gear graph $G_{0,14}$. We assign the colors $1,2,3, \ldots, 7$ to the edges of $G_{0,14}$ by using theorem 2 as shown in figure 7 .

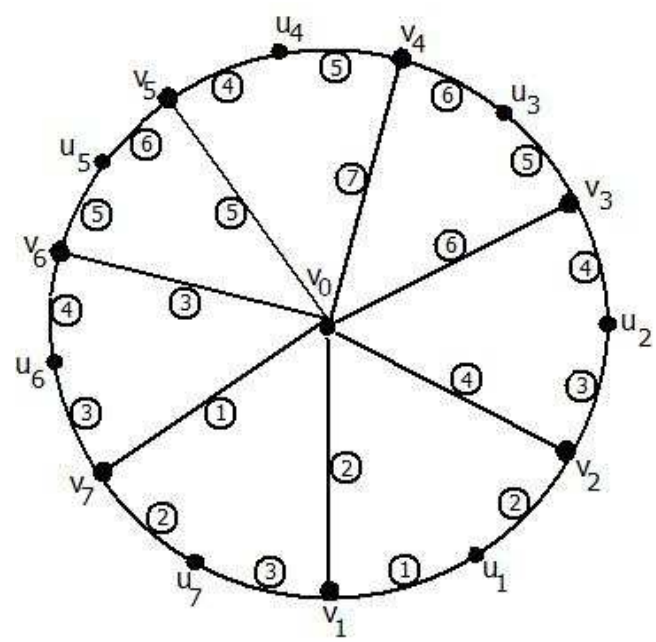

Figure 7: Gear graph $G_{0,14}$

This type of coloring, the gear graph $G_{0,14}$ satisfies the definition of interval edge coloring. Here $\chi_{i e}\left(G_{0,14}\right)=7$.

Illustration 6. Consider the gear graph $G_{0,16}$. We assign the colors $1,2,3, \ldots, 8$ to the edges of $G_{0,16}$ by using theorem 2 as shown in figure 8 . 
Interval Edge Coloring of Sudha Grid of Hexagons, Gear and Helm Graphs

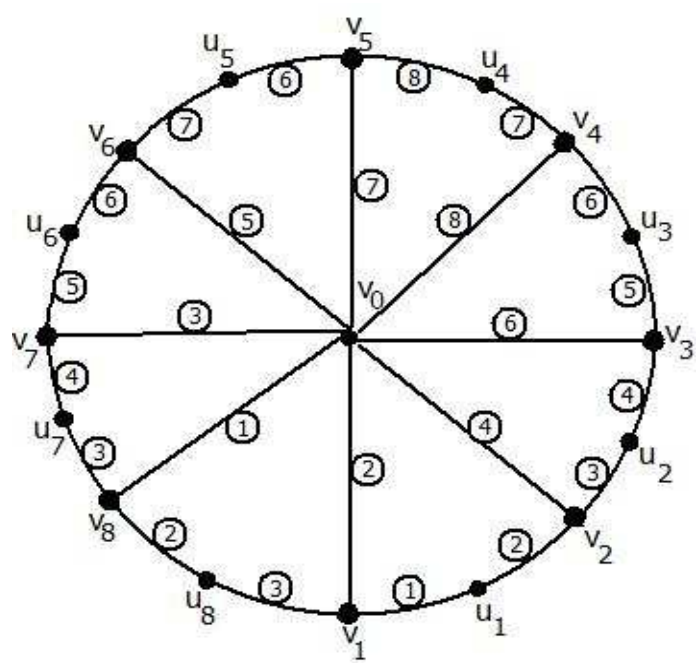

Figure 8: Gear graph $G_{0,16}$

This type of coloring, the gear graph $G_{0,16}$ satisfies the definition of interval edge coloring. Here $\chi_{i e}\left(G_{0,16}\right)=8$.

Theorem 3. The Helm graph $H_{0,2 n}$ admits interval edge coloring and its chromatic number of interval edge coloring is $n$.

Proof: The Helm graph $H_{0,2 n}$ has $2 n+1$ vertices and $3 n$ edges. The vertex set of $H_{0,2 n}$ is $V\left(H_{0,2 n}\right)=\left\{v_{0}, v_{i}, u_{i} / 1 \leq i \leq n\right\}$ and the edge set of $H_{0,2 n}$ is

$E\left(H_{0,2 n}\right)=\left\{v_{0} v_{i} / 1 \leq i \leq n\right\} \cup\left\{v_{i} u_{i} / 1 \leq i \leq n\right\} \cup\left\{v_{i} v_{i+1} / 1 \leq i \leq n-1\right\} \cup v_{n} v_{1}$ as shown in figure 9 .

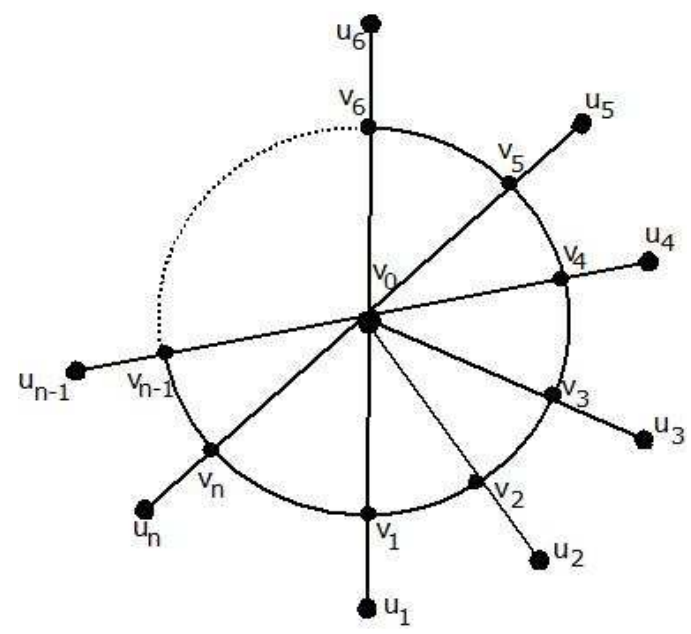

Figure 9: Helm graphs $H_{0,2 n}$

There are two cases : 
S. Sudha and G. M. Raja

Case (i): Let $n$ be odd.The function $f$ is defined as the coloring from the edges of $H_{0,2 n}$ to the set of colors (positive integers) $\{1,2,3, \ldots n\}$, as follows :

$$
\begin{aligned}
f\left(v_{0} v_{i}\right) & =\left\{\begin{array}{cc}
2 i, & 1 \leq i \leq \frac{n-1}{2} \\
2(n-i)+1, & \frac{n+1}{2} \leq i \leq n
\end{array},\right. \\
f\left(v_{i} u_{i}\right) & =\left\{\begin{array}{ll}
2(i-1), & 1<i<\frac{n-1}{2} \\
2(n-i)+3, & \frac{n+1}{2}<i \leq n
\end{array},\right. \\
f\left(v_{\frac{n-1}{2}} u_{\frac{n-1}{2}}\right) & =n, \\
f\left(\frac{v_{n+1}}{2} \frac{u_{n+1}}{2}\right) & =n-2, \\
f\left(v_{i} v_{i+1}\right) & = \begin{cases}2 i+1, & 1 \leq i<\frac{n-1}{2} \\
2(n-i), & \frac{n+1}{2} \leq i<n\end{cases} \\
f\left(v_{\frac{n-1}{2}} \frac{v_{n+1}}{2}\right)=n-3, \text { and } & f\left(v_{n} v_{1}\right)=4 .
\end{aligned}
$$

With this type of coloring, the Helm graph $H_{0,2 n}$ satisfies the definition of interval edge coloring and its chromatic number of interval edge coloring is $n$ for odd $n$.

Case (ii): Let $n$ be even.The function $f$ is defined as the coloring from the edges of $H_{0,2 n}$ to the set of colors (positive integers) $\{1,2,3, \ldots n\}$, as follows :

$$
\begin{gathered}
f\left(v_{0} v_{i}\right)=\left\{\begin{array}{c}
2 i, \quad 1 \leq i \leq \frac{n}{2}, \quad f\left(v_{i} u_{i}\right) \\
2(n-i)+1, \quad \frac{n}{2}<i \leq n
\end{array}, \quad \begin{array}{cc}
2(i-1), & 1<i \leq \frac{n}{2}+1 \\
2(n-i)+3, & \frac{n}{2}+1<i \leq n
\end{array},\right. \\
f\left(v_{1} u_{1}\right)=1, \quad 1 \leq i<\frac{n}{2}, \\
f\left(v_{i} v_{i+1}\right)= \begin{cases}2 i+1, & n \\
2(n-i), & \frac{n}{2}<i<n\end{cases} \\
f\left(v_{\frac{n}{2}} v_{\frac{n}{2}+1}\right)=
\end{gathered}
$$


Interval Edge Coloring of Sudha Grid of Hexagons, Gear and Helm Graphs and $\quad f\left(v_{n} v_{1}\right)=4$.

With this type of coloring, the Helm graph $H_{0,2 n}$ satisfies the definition of interval edge coloring and its chromatic number of interval edge coloring is $n$ for even $n$.

Illustration 7. Consider the Helm graph $H_{0,14}$. We assign the colors $1,2,3, \ldots, 7$ to the edges of $H_{0,14}$ by using theorem 3 , case (i), as shown in figure 10 .

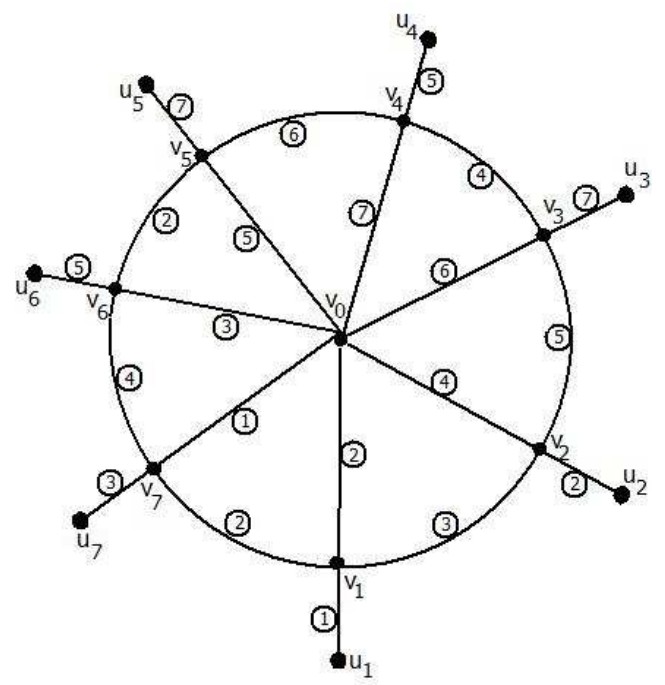

Figure 10: Helm graph $H_{0,14}$

This type of coloring, the Helm graph $H_{0,14}$ satisfies the definition of interval edge coloring. Here $\chi_{i e}\left(H_{0,2 n}\right)=7$.

Illustration 8. Consider the Helm graph $H_{0,16}$. We assign the colors $1,2,3, \ldots, 8$ to the edges of $H_{0,16}$ by using theorem 3, case (ii), as shown in figure 11 .

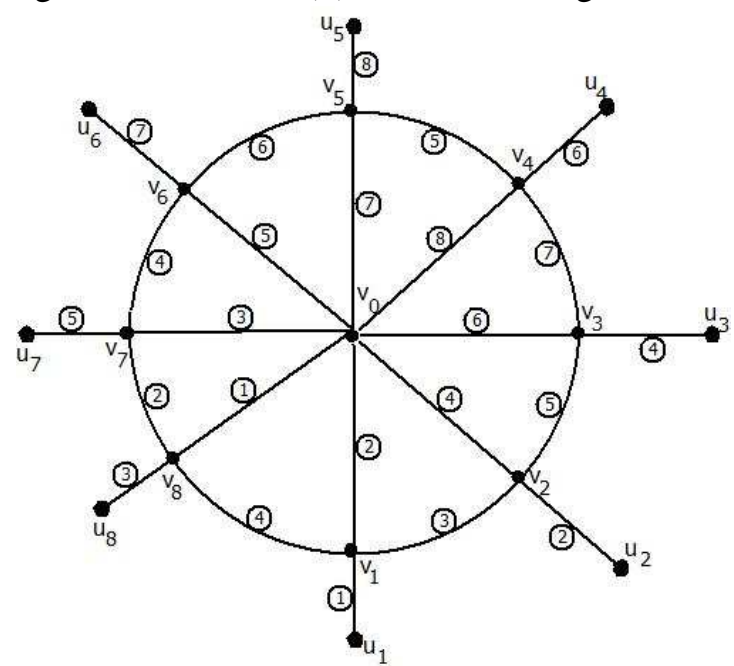

Figure 11: Helm graph $H_{0,16}$ 


\section{S. Sudha and G. M. Raja}

This type of coloring, the Helm graph $H_{0,16}$ satisfies the definition of interval edge coloring. Here $\chi_{i e}\left(H_{0,16}\right)=8$.

\section{Conclusion}

In this paper, we have proved that Sudha grid of hexagons $S_{h}(m, n)$ for admit interval edge coloring. Further, we have considered gear $G_{0,2 n}$ and Helm graph $H_{0,2 n}$ for all $\mathrm{n}$ and proved it admits interval edge coloring.

\section{REFERENCES}

1. A.S.Asratian and R.R.Kamalian, Interval coloring of edges of a multigraph, Applied Mathematics, Yerevan State University (in Russian), 5 (1987) 25-34.

2. K.Kaliraj and J.Vernold Vivin, On equitable coloring of Helm graph and gear graph, International Journal of Mathematics Combinatory, 2 (2010) 32-37.

3. R.R.Kamalian, Interval colorings of complete bipartite graphs and trees, Preprint of the Computing Centre of the Academy of Science of Armenia (in Russian), 1989.

4. R.R.Kamalian, On a number of colors in cyclically interval edge colorings of trees, Research Report LiTHMAT-R-2010/09-SE, Linkoping University, 2010.

5. R.R.Kamalian, On a number of colors in cyclically interval edge colorings of simple cycles, Open Journal of Discrete Mathematics, 3 (2013) 43-48.

6. S.Meena and K.Vaithilingam, Prime labeling for some Helm related graphs, IJIRSET, 2 (2013).

7. P.A.Petrosyan, Interval edge coloring of complete graphs and $\mathrm{n}$ dimensional cubes, Discrete Mathematics, 310 (2010) 1580-1587.

8. A.Saha, M.Pal and T.K.Pal, Selection of programme slots of television channels for giving advertisement: A graph theoretic approach, Information Sciences, 177 (12) (2007) 2480-2492.

9. K.Sathish Kumar, N.Gnanamalar David and K.G.Subramanian, Graphs and power dominator colorings, Annals of Pure and Applied Mathematics, 11 (2016) 67-71.

10. S.Sudha and V.Kanniga, Star-in-coloring of Benzenoid graphs (coronene series) and grid graphs, Asian Journal of Current Engineering and Mathematics, 4 (2015).

11. S.Sudha and G.M.Raja, Interval edge coloring of grid graphs and equitable interval edge coloring of grid of diamonds and prism graphs, International Journal of Scientific and Innovative Mathematical Research, 2 (2014) 408-417.

12. S.K.Vaidya and N.H.Shah, Prime cordial labeling of some wheel related graphs, Malaya Journal of Mathematics, 4 (2013) 148-156. 